\title{
In Vitro Biocompatibility Test of Multi-layered Plasmonic Substrates with Flint Glasses and Adhesion Films
}

\author{
Nak-Hyeon Kim¹, Kyung Min Byun ${ }^{1 *}$, Seoyoung Hwang², Yena Lee², and Sang Beom Jun ${ }^{2,3}$ \\ ${ }^{1}$ Department of Biomedical Engineering, Kyung Hee University, Yongin 446-701, Korea \\ ${ }^{2}$ Department of Electronics Engineering, Ewha Womans University, Seoul 120-750, Korea \\ ${ }^{3}$ Department of Brain and Cognitive Sciences, Ewha Womans University, Seoul 120-750, Korea
}

(Received January 17, 2014 : revised February 25, 2014 : accepted February 25, 2014)

\begin{abstract}
Since in vitro neural recording and imaging applications based on a surface plasmon resonance (SPR) technique have expanded dramatically in recent years, cytotoxicity assessment to ensure the biosafety and biocompatibility for those applications is crucial. Here, we report the cytotoxicity of the SPR substrate incorporating a flint glass whose refractive index is larger than that of a conventional crown glass. A high refractive index glass substrate is essential in neural signal detection due to the advantages such as high sensitivity and wide dynamic range. From experimental data using primary hippocampal neurons, it is found that a lead-based flint glass is not appropriate as a neural recording template although the neuron cells are not directly attached to the toxic glass. We also demonstrate that the adhesion layer between the glass substrate and the gold film plays an important role in achieving the substrate stability and the cell viability.
\end{abstract}

Keywords: Biocompatibility, Surface plasmons, Flint glasses, In vitro neuron cell culture

OCIS codes : (160.2750) Glass and other amorphous materials; (240.6680) Surface plasmons

\section{INTRODUCTION}

Extracellular measurement of neural activity is a key technology for research on neural interfaces such as cultured neural networks. While electrical recording based on microelectrode arrays has been widely used, an electrical method often suffers from stimulation artifacts when an electrical signal is applied [1]. Moreover, the electrodes should be placed close to the neuronal cell body for increasing the efficacy of the interface and excluding the noise sources. On the other hand, as demonstrated lately [2], alternative optical approaches have drawn tremendous interest due to their advantages such as noninvasive and artifact-free monitoring and high spatial resolution. Although voltagesensitive fluorescence dyes allow direct visualization of a membrane potential change, optical staining has fundamental drawbacks of cell toxicity, a time-consuming labeling process, and photobleaching [3]. In recent years, a surface plasmon resonance (SPR) technique has been introduced as intrinsic and label-free optical recording method.
In principle, an SPR biosensor measures a refractive index change at a dielectric-metal interface. As SPR detection is rapid, quantitative, and sensitive, it has been utilized to monitor the kinetics of surface-immobilized interactions [4]. Those properties allow the SPR system to sense the neural activities because action potential is accompanied by a small change in membrane-localized refractive index. In the previous studies, we verified the effectiveness of SPR-based optical detection in both in vitro and in vivo neural recordings for isolated rat sciatic nerve and somatosensory cortex $[5,6]$. However, the following key issues should be addressed for practical use of SPR-based neural recording: 1) What are requirements of the glass substrate to provide better performance and to ensure biocompatibility in SPR sensing? We demonstrate numerically whether glass material of a high refractive index is useful as an SPR substrate in terms of sensitivity and dynamic range in measuring neural signals [7]. In particular, a leaded flint glass cannot assure the biosafety of culture environments even though the neuron cells are not directly attached to the toxic glass, but

\footnotetext{
*Corresponding author: kmbyun@khu.ac.kr

Color versions of one or more of the figures in this paper are available online.
} 
separated from it via a gold film and an adhesion layer. 2) What are the effects of thin metallic films on the cell viability? Gold is usually biocompatible and inert, but an exposure to a titanium or chromium adhesion layer might be injurious to living cells.

Among various instrumental SPR sensing platforms, the most widely used substrate includes BK7 glass, which is known as a biocompatible crown glass. Crown glass has a low refractive index and is produced from alkali-lime silicates containing potassium oxide. On the other hand, flint glass has a relatively high refractive index ranging from 1.46 to 2.00. To obtain a higher refractive index, several additives such as lead oxide, titanium oxide, or zirconium oxide are usually contained in the flint glass. In terms of cytotoxicity, lead-based glass substrates and adhesion metal layers may have harmful effects on the cultured cells $[8,9]$. In other words, while the neurons are cultured onto a gold film, the stack configuration of SPR templates including toxic materials can exert unidentified adverse effects on the neuron cells.

In this study, we, for the first time to our knowledge, investigate whether the viability of cultured neuron cells can be affected by the substrate materials. To evaluate the cellular cytotoxicity in a quantitative way, we count the number of primary hippocampal neurons grown on various SPR substrates, fabricated by combining three different glasses of BK7, SF10, and NSF10 with two adhesion metal films of chromium and titanium. It is expected that our study will serve as the first step to design an optimal configuration of the SPR substrate for improving the biocompatibility of an in vitro neural recording system.

\section{EXPERIMENTAL DETAILS}

\subsection{Preparation of SPR Substrates}

Three kinds of glasses were purchased from Marienfeld GmbH (Lauda-Königshofen, Germany) for BK7, Korea ElectroOptics (Bucheon, Korea) for SF10, and Schott (Mainz, Germany) for NSF10. While the Marienfeld BK7 glass containing $10 \%$ boric oxide has been frequently used as a substrate for cell cultures due to low alkali content, there have been few reports on the application of flint glasses such as SF10 and NSF10 to in vitro studies on live cells. Refractive indices of the glasses are, respectively, $n_{\mathrm{BK} 7}=$ 1.52 and $n_{\mathrm{SF} 10}=n_{\mathrm{NSF} 10}=1.72$ at $\lambda=633 \mathrm{~nm}$. All the glass slides have a square shape of $20 \mathrm{~mm} \times 20 \mathrm{~mm}$. After cleaning a glass with piranha solution of $\mathrm{H}_{2} \mathrm{SO}_{4}: \mathrm{H}_{2} \mathrm{O}_{2}=$ $3: 2$, the SPR template was produced by sputtering a 45-nm thick gold film onto the glass after an evaporation of a 5-nm thick adhesion layer of chromium or titanium as shown in Fig. 1. The fabricated samples were cleaned sequentially in acetone and $70 \%$ ethanol for $10 \mathrm{~min}$ in a sonication bath and then rinsed with distilled water and dried by blowing nitrogen. We also fabricated SPR samples without any adhesion layer to explore the influence of chromium or titanium on the cellular toxicity.

\subsection{Cell Culture}

Primary hippocampal neurons were obtained by referring to Ref. [10]. Whole brains were isolated from fetuses of embryonic day 17 Sprague-Dawley rats (Samtako, Osan, Korea) and were placed in sterile buffered saline solution (BSS). Hippocampi were dissected from the separated cerebral hemispheres, placed in ice-cold BSS and incubated in $0.25 \%$ trypsin (Sigma, St. Louis, MO, USA) for $15 \mathrm{~min}$ at $37^{\circ} \mathrm{C}$. Digested hippocampi were dissociated three times for $5 \mathrm{~min}$ in BSS at room temperature by triturating with fire-polished glass pipettes. Neurons were seeded at the density of about 300 cells $/ \mathrm{mm}^{2}$ on the SPR samples in minimal essential medium (MEM) supplemented with $10 \%$ horse serum and $0.1 \%$ pyruvic acid (Invitrogen, Carlsbad, CA). After $4 \mathrm{hr}$ incubation, the MEM medium was replaced with serum-free Neurobasal media (Invitrogen, Carlsbad, CA, USA) supplemented with B27 (Invitrogen) and GlutaMAX (Invitrogen). During the experiments, the media was replaced with fresh every 3 days. Cultures were maintained at $37^{\circ} \mathrm{C}$ in a $5 \% \mathrm{CO}_{2}, 95 \%$ air humidified atmosphere.

\subsection{Cell Counting and Data Analysis}

A hemacytometer was used to obtain the initial neuronal cell density of 300 cells $/ \mathrm{mm} 2$. After seeding neurons on the surface, the cells were imaged using an inverted microscope (IX71, Olympus, Japan) for 3 weeks. Only the cell bodies with axons and dendrites were counted as the number of cells during in vitro cell culture. The number of cells per unit area of $440 \mu \mathrm{m} \times 273 \mu \mathrm{m}$ was counted 3 times in different sites for each sample after replacement of the media which were replaced every 3 days.

\subsection{Transfer-matrix Method (TMM)}

The analytical solution of reflectance $R$ is represented

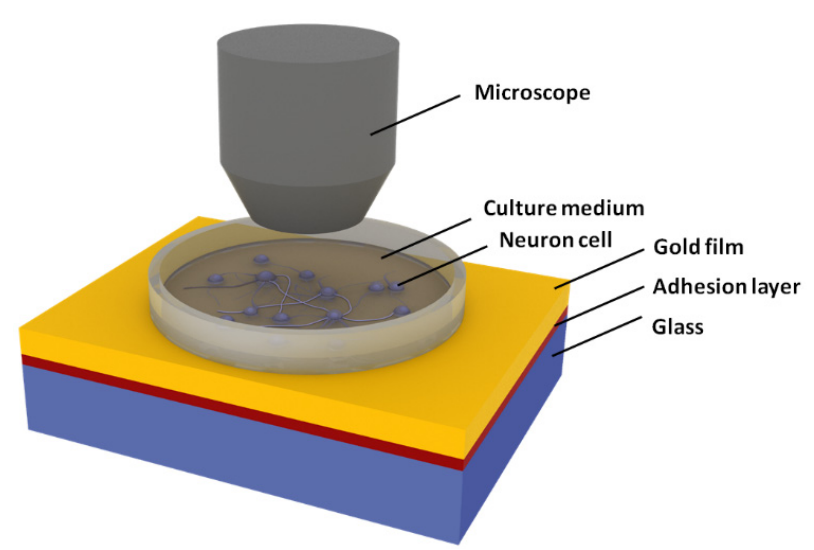

FIG. 1. Schematic of the SPR substrate for in vitro neural recording. A 45-nm-thick gold film is deposited on a glass substrate via adhesion layer with a thickness of $5 \mathrm{~nm}$. The substrate is modified for use as a culture chamber using a Teflon ring filled with a neurobasal media with serum. After seeding neurons on the substrate surface, the neuron cells are imaged using an inverted microscope for 3 weeks. 
by a $2 \times 2 M$-matrix, which is a serial product of the interface matrix $I_{j k}(j=0,1,2$ and $k=j+1)$ and the layer matrix $L_{j}$ as follows:

$$
R=\left|\frac{M_{12}}{M_{22}}\right|^{2}
$$

where

$$
\begin{aligned}
M & =\left[\begin{array}{ll}
M_{11} & M_{12} \\
M_{21} & M_{22}
\end{array}\right]=I_{01} L_{1} I_{12} L_{2} I_{23} L_{3} I_{34}, \\
I_{j k} & =\left[\begin{array}{cc}
1 & r_{j k} \\
r_{j k} & 1
\end{array}\right], \\
\text { and } L_{j} & =\left[\begin{array}{cc}
e^{i k_{z j} d_{j}} & 0 \\
0 & e^{-i k_{z j} d_{j}}
\end{array}\right] .
\end{aligned}
$$

Here, $r_{j k}, k_{z}$, and $d_{j}$ represent the Fresnel reflection coefficient, wave vector in the z-direction, and the thickness of $j$ th layer, respectively. $r_{j k}$ and $k_{z j}$ are given by

$$
\begin{aligned}
& r_{j k}=\frac{\left(\frac{k_{z j}}{\varepsilon_{j}}-\frac{k_{z k}}{\varepsilon_{k}}\right)}{\left(\frac{k_{z k}}{\varepsilon_{j}}-\frac{k_{z k}}{\varepsilon_{k}}\right)} \text { and } \\
& k_{z j}=\sqrt{\varepsilon_{j}\left(\frac{\omega}{c}\right)^{2}-k_{x}^{2}} \text { with } k_{x}=\sqrt{\varepsilon_{0}} \frac{\omega}{c} \sin \theta_{0}
\end{aligned}
$$

where $\omega$ is the angular frequency, $\theta_{0}$ is the incidence angle, $c$ is the speed of light in free-space, and $\varepsilon_{0}$ is the relative permittivity of a glass substrate. Details of our TMM algorithm can be found elsewhere [11].

\section{RESULTS AND DISCUSSION}

First of all, we numerically demonstrate whether the SPR sensing performance for neural recording can be improved by a high refractive index glass, when compared against a conventional crown glass. Using the TMM, we determine the reflectance characteristics of a 4-layer SPR system consisting of glass substrate, chromium adhesion layer, gold film, and cell culture medium [12]. Based on the fact that an effective refractive index of a single cell is known to be about 1.37 [13], as neural activities are accompanied by small changes in the cellular volume and the membrane-localized liquid phase, the refractive index of the solution is assumed to be varied in the range from 1.36 to 1.38 . Mechanical fluctuations such as cell swelling and shrinkage during the period of action potential also contribute to the refractive index change. The relative permittivities $\varepsilon=(n, k)$ of crown and flint glasses, $5-\mathrm{nm}$ thick chromium and 45-nm thick gold films are set to be
$(1.52,0),(1.72,0),(3.48,4.36)$, and $(0.18,3.00)$, respectively, at $\lambda=633 \mathrm{~nm}$ [14].

The calculated SPR curves are shown in Fig. 2 for two glass substrates of low and high refractive indices $n$. When a refractive index of the surrounding solution increases, clear advantages in the latter case are evident. The resonance band of a high refractive index glass is sharper and deeper, implying superiority in detection sensitivity and signal-to-noise ratio. On the other hand, the glass substrate of a low refractive index gives a broad and shallow resonance peak. In particular, a higher SPR angle over $80^{\circ}$ makes practical implementation extremely difficult and dynamic range in measurement very narrow. As a result, the choice of a higher refractive index glass is appropriate for monitoring the neural signals if its biocompatibility is verified. Recently, a crown glass with a high refractive index, for example, LAK34, has been developed. However, owing to its low acid resistance even in weak acid, an acidic solution-based surface cleaning process to remove organic residues may cause severe damages to the glass substrate.

Hence, in the following section, we intend to demonstrate the possibility of flint glass for optical neural recording as an alternative to a crown glass. We perform a biosafety test using two different flint glasses of SF10 and NSF10 with an equal refractive index. While a flint glass generally contains lead oxide, NSF10 is a lead-free glass whose lead compound is replaced with other additives such as titanium dioxide and zirconium dioxide without altering the optical properties.

Figure 3 shows microscope images of the cultured neuron cells at 12 days in vitro (DIV). While the results are not shown, we first observed the growth and position of neurons at 3 DIV and each neuron started to make small connections with neighboring cells. At 12 DIV, while the BK7 and

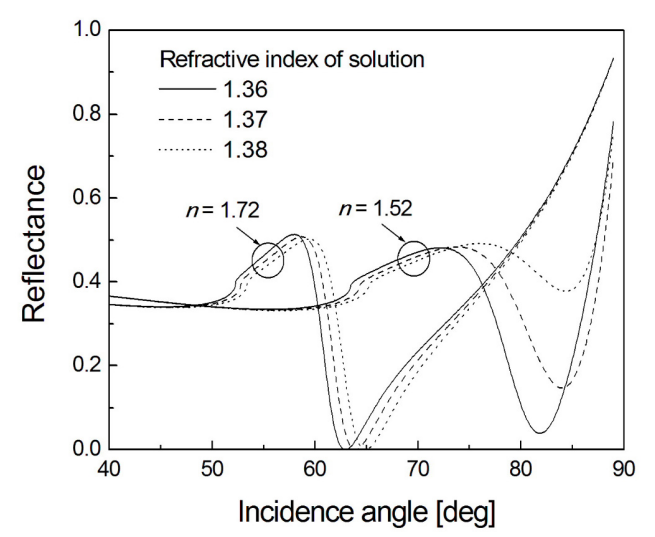

FIG. 2. Calculated SPR curves for two glass substrates of low and high refractive indices. The curves are computed under the incidence of $\lambda=633 \mathrm{~nm}$ and the scanning angle resolution of $0.01^{\circ}$. When a positive variation in refractive index of the solution occurs, the resonance angle is increasing and the resonance dip becomes shallower, especially for $n=$ 1.52 (i.e., BK7 glass), while the shape of SPR curves for $n=$ 1.72 does not change significantly. 
NSF10 glasses show greatly concentrated cell bodies and dendrites of neurons, no cellular structure is found at the SF10 glass, which implies that SF10 glass inhibits neuronal

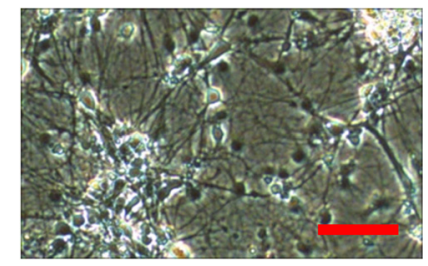

(a) $\mathrm{BK} 7 / \mathrm{Cr} / \mathrm{Au}$

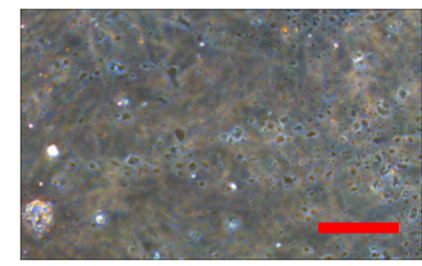

(c) $\mathrm{SF} 10 / \mathrm{Cr} / \mathrm{Au}$

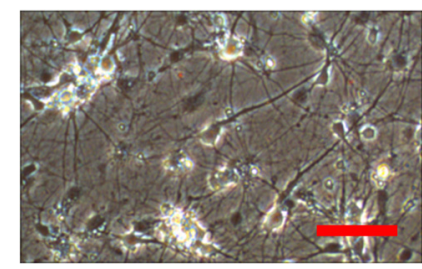

(e) $\mathrm{NSF} 10 / \mathrm{Cr} / \mathrm{Au}$

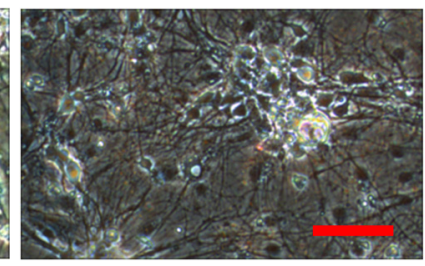

(b) $\mathrm{BK} 7 / \mathrm{Ti} / \mathrm{Au}$

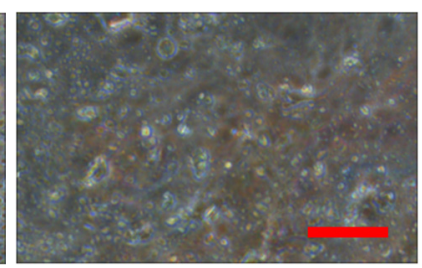

(d) SF10/Ti/Au

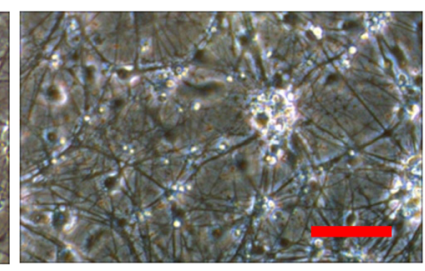

(f) $\mathrm{NSF} 10 / \mathrm{Ti} / \mathrm{Au}$
FIG. 3. Microscope images of the cultured hippocampal neuron cells at 12 DIV. The results in (a) and (b) show the cultured neurons for conventional BK7 glass substrates as a control. In the cases of (c) and (d), cell death is observed at a whole region of the SPR substrate based on a leaded SF10 glass. For lead-free NSF10 glass substrate, dense and multilayered axons and dendrites in the cell bodies are exhibited, as shown in (e) and (f), which resembles the results of (a) and (b). Scale bar $=100 \mu \mathrm{m}$.

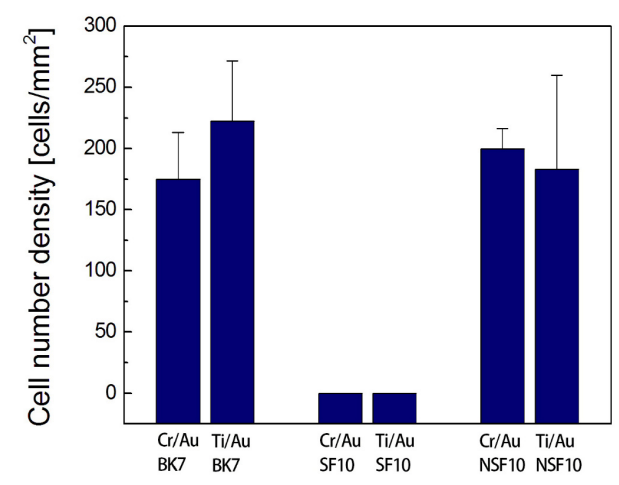

FIG. 4. Quantitative analysis of the effect of glass and adhesion materials on the cell number density at 12 DIV. The cell density is determined by counting the cell number in 440 $\mu \mathrm{m} \times 273 \mu \mathrm{m}$ regions. In an initial cell density of 300 cells $/ \mathrm{mm}^{2}$, cell number is measured 3 times in different sites for each sample. Throughout, error bars indicate the standard deviation of the mean. cell growth by releasing toxic materials that can inactivate neural activities. For BK7 and NSF10 glass substrates, a number of neurons are still alive after 18 DIV and dense and multilayered axons and dendrites in the cell bodies are exhibited.

It is also interesting to note that there is no significant difference in overall trends of cell survival, cell morphology, and neurite elongation between the substrates with chromium and titanium. Adhesion layers might have no adverse influence on the metabolic activity of cultured cells because a 5-nm thick layer is too thin to affect the cell viability. The cell number densities obtained are, respectively, $175 \pm 38$ cells $/ \mathrm{mm}^{2}$ for $\mathrm{BK} 7 / \mathrm{Cr} / \mathrm{Au}, 222 \pm 49$ cells $/ \mathrm{mm}^{2}$ for BK7/ $\mathrm{Ti} / \mathrm{Au}, 200 \pm 17 \mathrm{cells} / \mathrm{mm}^{2}$ for $\mathrm{NSF} 10 / \mathrm{Cr} / \mathrm{Au}$, and $183 \pm$ 77 cells $/ \mathrm{mm}^{2}$ for NSF10/Ti/Au substrate, as presented in Fig. 4. While multiple sites in a given sample are measured by spatial translation to ensure consistency and thereby to reduce variation, the standard error is relatively large because the cell distribution is not perfectly uniform on the gold substrate.

As a next set of experiments to verify that SF10 glass is harmful, neuron cells are directly cultured on a glass substrate without metal films. Cell images at 15 DIV in Fig. 5 show the cytotoxic effect caused by lead-based

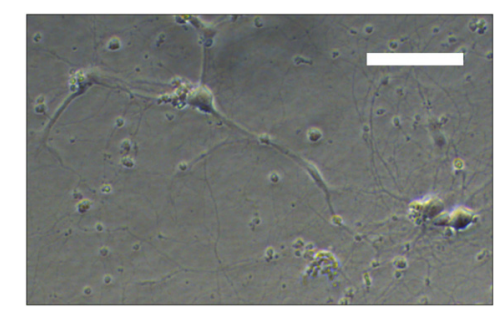

(a) BK7

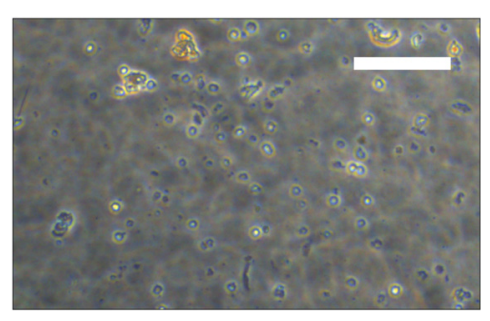

(b) SF10

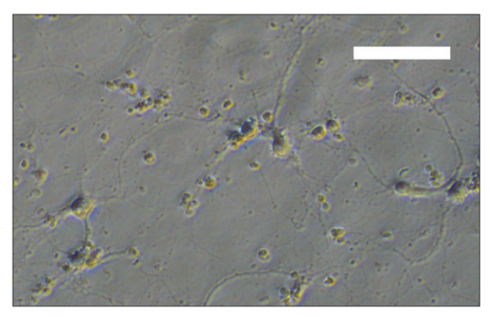

(c) NSF10

FIG. 5. Microscope images when neuron cells are directly cultured on a glass substrate without metal films. While the cell images obtained at 15 DIV clearly show the cytotoxic effect caused by lead-based SF10 glass substrate, the other glasses have no toxic effect on the neuron cell culture. Scale bar $=100 \mu \mathrm{m}$. 
SF10 glass. Contrary to the cultures in BK7 and NSF10 glasses, a rapid neuronal cell death occurs after exposure to SF10 glass. It is attributed to the fact that lead causes the axons of nerve cells to degenerate and lose their myelin coats. It is also known that lead enters the neurons via voltage-sensitive calcium channels and induces mitochondrial dysfunction, which can lead to a release of cytochrome $\mathrm{C}$, activation of a variety of caspases and cleavage of downstream death effector proteins, and ultimately results in apoptotic cell death [15]. In short, the existence of toxic materials in glass substrate should be avoided during the culture for improving the cell viability.

Another remaining issue of interest is the role of adhesion layers in neuronal cell culture. Thin transition metal films, such as chromium, titanium, and tungsten are generally employed as an adhesion promoter between a metal film and a glass substrate. They not only improve the adhesion, but enhance the stability of the noble metal layer. If the adhesion film is not used, the metal film supporting plasmon excitation can often be peeled off and damaged by the cleaning processes. In this experiment, we compare the cell number density of SPR substrates with 5-nm thick chromium adhesion with the cases in which a gold film is directly deposited on a glass substrate. In Fig. 6 , a higher cell number is obtained in the presence of chromium for both BK7 and NSF10 glasses. At 12 DIV, the cell number densities are determined to be $120 \pm 65$ cells $/ \mathrm{mm}^{2}$ for BK7/Au and $102 \pm 46$ cells $/ \mathrm{mm}^{2}$ for NSF10/ $\mathrm{Au}$ substrates, which are approximately $50 \sim 60 \%$ of the cell survival for SPR substrates with a chromium layer. The substrate instability is responsible for poor attachment of neuron cells and this may contribute to a smaller cell density and a larger standard error. On the other hand, it has been reported in several in vitro studies that chromium

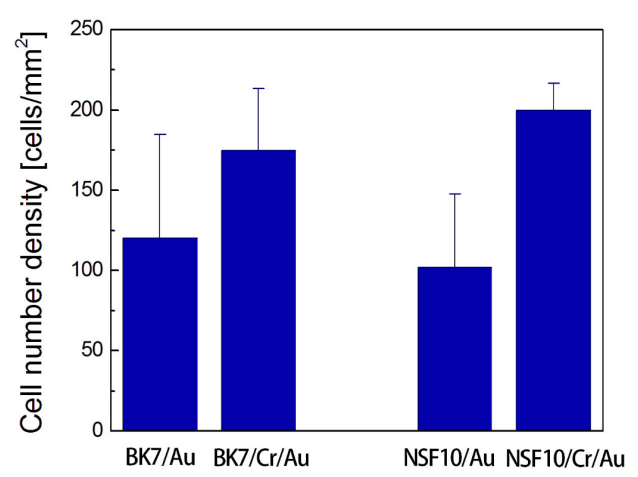

FIG. 6. Quantitative analysis of the effect of adhesion layer on the cell number density at $12 \mathrm{DIV}$. The cell density is determined by counting the cell number in $440 \mu \mathrm{m} \times 273 \mu \mathrm{m}$ regions. In an initial cell density of 300 cells $/ \mathrm{mm}^{2}$, cell number is measured 3 times in different sites for each sample. The SPR templates without an adhesion layer show a relatively smaller cell density due to the substrate unstability which can prevent the neuron cells from being attached to the substrate efficiently. thicker than $50 \mathrm{~nm}$ can inhibit the metabolic response of cultured cells such as stem cells [8], osteoblastic cells [9], macrophages [16]. In particular, since a thick adhesion film can degrade the performance of SPR-based neural recording system due to a large imaginary part of its complex dielectric constant, careful substrate design is required for an efficient neuron cell culture.

\section{CONCLUSION}

In this study, we demonstrate that flint glass substrates of a high refractive index have a potential for improving SPR-based neural recording system due to significant advantages in detection sensitivity, signal-to-noise ratio, and dynamic range compared to a conventional crown glass. Based on the cytotoxicity test performed to verify the possibility of applying the flint glass to in vitro neuronal cell culture, we find that SF10 glass including lead oxide shows harmful effects to the cultured neurons, whereas another lead-free flint glass of NSF10 is not problematic. The lead-mediated cytotoxicity is inevitable even when the neuron cells are separated from SF10 glass via thin adhesion layer and gold film. It is also found that the adhesion layer of chromium and titanium plays an important role in achieving the substrate stability. The cell density of surviving neurons on gold films deposited with a metallic adhesion layer was approximately twice that of the films without the adhesion layer due to the increased mechanical stability. As a result, by applying a lead-free flint glass of a high refractive index and a thin adhesion film to SPR substrate, we may expect to accomplish an improved performance in culturing the neuron cells and recording their signals.

\section{ACKNOWLEDGMENT}

We acknowledge the support of Korea Science and Engineering Foundation (KOSEF) grant funded by the Korean government (MEST) (2011-0029485). This work was also supported by NSF-2013R1A1A1A05011990 and by MSIP as Global Frontier Project (CISS-2013072261).

\section{REFERENCES}

1. F. O. Morin, Y. Takamura, and E. Tamiya, "Investigating neuronal activity with planar microelectrode arrays: Achievements and new perspectives," J. Biosci. Bioeng. 100, 131-143 (2005).

2. G. Cui, S. B. Jun, X. Jin, M. D. Pham, S. S. Vogel, D. M. Lovinger, and R. M. Costa, "Concurrent activation of striatal direct and indirect pathways during action initiation," Nature 494, 238-242 (2013).

3. T. J. Ebner and C. Gang, "Use of voltage-sensitive dyes and optical recordings in the central nervous system," Prog. Neurobiol. 46, 463-506 (1995). 
4. J. Homola, S. S. Yee, and G. Gauglitz, "Surface plasmon resonance sensors: Review," Sens. Actuators B 54, 3-15 (1999).

5. S. A. Kim, K. M. Byun, J. Lee, J. H. Kim, D.-G. A. Kim, H. Baac, M. L. Shuler, and S. J. Kim, "Optical measurement of neural activity using surface plasmon resonance," Opt. Lett. 33, 914-916 (2008).

6. S. A. Kim, S. J. Kim, H. Moon, and S. B. Jun, "In vivo optical neural recording using fiber-based surface plasmon resonance," Opt. Lett. 37, 614-616 (2012).

7. R. Micheletto, K. Hamamoto, T. Fujii, and Y. Kawakam, "Tenfold improved sensitivity using high refractive-index substrates for surface plasmon sensing," Appl. Phys. Lett. 93, 174104 (2008).

8. M. Stiehler, M. Lind, T. Mygind, A. Baatrup, H. Li, M. Foss, F. Besenbacher, M. Kassem, and C. Bünger, "Morphology, proliferation, and osteogenic differentiation of mesenchymal stem cells cultured on titanium, tantalum, and chromium surfaces," J. Biomed. Mater. Res. Part A 86A, 448-458 (2008).

9. M. J. Allen, B. J. Myer, P. J. Millett, and N. Rushton, "The effects of particulate cobalt, chromium and cobaltchromium alloy on human osteoblast-like cells in vitro," J. Bone Joint Surg. Am. 79B, 475-482 (1997).
10. S. H. Jeong, S. B. Jun, J. K. Song, and S. J. Kim, "Activitydependent neuronal cell migration induced by electrical stimulation," Med. Biol. Eng. Comput. 47, 93-99 (2009).

11. S. H. Choi and K. M. Byun, "Investigation on an application of silver substrates for sensitive surface plasmon resonance imaging detection,” J. Opt. Soc. Am. A 27, $2229-2236$ (2010).

12. A. Yariv and P. Yeh, Optical Waves in Crystals: Propagation and Control of Laser Radiation (Wiley, New York, USA, 1984).

13. B. Rappaz, P. Marquet, E. Cuche, Y. Emery, C. Depeursinge, and P. J. Magistretti, "Measurement of the integral refractive index and dynamic cell morphometry of living cells with digital holographic microscopy," Opt. Express 13, 93619373 (2005).

14. E. D. Palik, Handbook of Optical Constants of Solids (Academic, New York, USA, 1985).

15. T. I. Lidsky and J. S. Schneider "Lead neurotoxicity in children: Basic mechanisms and clinical correlates," Brain 126, 5-19 (2003)

16. I. Catelas, A. Petit, D. J. Zukor, and O. L. Huk. "Cytotoxic and apoptotic effects of cobalt and chromium ions on J774 macrophages-Implication of caspase-3 in the apoptotic pathway," J. Mater. Sci. Mater. Med. 12, 949-953 (2001). 